\title{
Impact de la ventilation mécanique et des méthodes de ventilation sur la perception de vie et de santé de malades atteints de dystrophie musculaire de Duchenne
}

Ghilas Boussaid, Christian Réveillère

Résumé

L'objectif de cette étude a été d'évaluer les avantages et inconvénients de la dépendance à un ventilateur perçus par des sujets atteints de dystrophie musculaire de Duchenne (DMD), sur leur vie en général (selon trois dimensions : la vie professionnelle, les loisirs ou vacances, et la vie de famille), et sur leur santé en particulier [1]. Méthodes : il s'agissait d'une enquête transversale réalisée par le biais d'un questionnaire adressé directement aux personnes atteintes de DMD suivies par le département des actions auprès des familles de l'AFM-Téléthon. Sur 663 sujets répondeurs, 190 avaient une ventilation mécanique (VM), 110 (57\%) étaient ventilo-dépendants, dont 47 (43\%) étaient en ventilation non invasive en continu (VNIc) et 63 (57 \%) en ventilation invasive (VI). Les sujets ont été regroupés en fonction de leur dépendance à la VM, nocturne versus dépendant, et en fonction du type de VM utilisé : ventilation non invasive (VNI) versus VI. Près de $85 \%$ de cette population de sujets ventilés était globalement satisfaite des soins médicaux et ce, quel que soit le niveau de dépendance ou le type de VM. Indépendamment du niveau de $\mathrm{VM}$ et de son mode opératoire, les sujets gastrostomisés ont nécessité plus fréquemment des consultations en urgence pour des troubles digestifs $(22,5 \%$ vs $4,6 \%, p=0,001)$. Aucun lien significatif entre la méthode de VM et la difficulté à parler n'a été observé, les problèmes de phonation étant bien compensés y compris pour la VI. En revanche, les sujets, quels que soient le niveau de dépendance et le type de VM, avaient une perception des difficultés de vie sociale quasi équivalente. Nous sommes là face à un impact général sur la vie familiale, les loisirs et les vacances qui s'est révélé accentué par l'évolution de la maladie (de 76,6\% à $92,3 \%$ selon le niveau de dépendance). Autre effet de l'évolution, les sujets DMD qui étaient dépendants en continu du ventilateur ont rapporté plus de douleurs, de problèmes digestifs et une qualité moindre de sommeil (problèmes d'insomnie et de somnolence diurne) et ce, en particulier chez les sujets en VI. Enfin, si cette population avait un niveau élevé d'anxiété $(39,6 \%)$ qui pouvait affecter la qualité du sommeil, la ventilation continue et la trachéostomie ne le majoraient pas. Il en va de même pour le risque de dépression (dépressivité) dont le niveau demeure élevé mais dans une relative moindre mesure $(16,7 \%)$.

\section{Commentaire}

Dans la DMD, l'espérance de vie s'est considérablement améliorée depuis la prise en charge de l'insuffisance respiratoire par la ventilation mécanique. Par le passé, lorsque les sujets devenaient dépendants de la VM, une ventilation invasive était presque systématiquement proposée par les équipes médicales. Actuellement, avec les progrès technologiques des assistances respiratoires mécaniques, y compris pour l'aide à la toux, la VNIc, qui peut le cas échéant nécessiter l'utilisation d'une pièce buccale (type pipette) pendant la journée, constitue une alternative à la VI. Le choix entre VNIc ou VI pouvant dépendre principalement des préférences du patient, il a été important de recueillir les jugements de sujets DMD ventilo-dépendants, qui avaient précédemment fait leur choix et expérimenté une VNI ou une VI. En ce sens, cette enquête rétroactive pourra être utile pour les sujets DMD plus jeunes. Au terme de cette étude, il ressort que la VI n'a pas d'impact majeur sur la perception de la vie chez les sujets DMD, en dehors du sommeil. Les problèmes d'insomnie peuvent sans doute s'expliquer par un changement de positionnement régulier dans le lit effectué par un tiers. L'utilisation de nouveaux matelas dynamiques s'est avérée bénéfique dans la prévention des lésions tissulaires et l'amélioration de la qualité de vie (QDV) des sujets [2,3]. Sur un tout autre plan, il ne semble pas y avoir plus de difficultés phonatoires chez les patients en VI. Résultat intéressant car on sait combien la communication est un enjeu majeur pour les sujets en VI et combien elle peut impacter la qualité de vie. Ce bon résultat est à mettre en lien avec le fait que la majorité des sujets ont bénéficié de soins spécifiques de phonation. Ceux-ci permettent aux sujets sous VI de parler pendant l'inspiration et l'expiration. Elle permet aussi d'augmenter la pression sous-glottique ce qui est de nature à améliorer l'intensité de la voix. 
Des difficultés dans la vie sociale sont soulignées pour toute cette population. Sans doute cette tendance au retrait social contraint est un effet de l'évolution de la maladie inductrice de dépendance fonctionnelle et respiratoire, de douleurs, ou de problèmes digestifs. Ceci induit un état d'inconfort et de vulnérabilité physique et psychologique (niveaux non négligeables d'hyperanxiété et de dépressivité) qui gêne la participation sociale du patient et sans doute aussi celle de son entourage.

Impact of mechanical ventilation methods on life perception for patients with Duchenne de Boulogne

\section{LIENS D'INTÉRÊT}

Les auteurs déclarent n'avoir aucun lien d'intérêt concernant les données publiées dans cet article.

\section{RÉFÉRENCES}

1. Boussaïd G, Stalens C, Devaux C, Segovia-Kueny S, Lofaso F, Réveillere C. Impact of mechanical ventilation methods on the life perception for patients with Duchenne de Boulogne: French cross-sectional survey. Respiratory Care 2020 (sous presse).

2. Chamanga E, Butcher A. An evaluation of a novel alternating mattress and cushion technology. Br J Community Nurs 2016 ;

21 (suppl 3) : S25-31.

3. Newton H. Evaluating a pressure-redistribution mattress replacement system. Br J Nurs 2014 ; 23 (suppl 20) : S30-5.

\begin{tabular}{|c|c|c|}
\hline \multicolumn{2}{|l|}{ MYOBASE "'?" } & AFMTELETHON "'? \\
\hline \multicolumn{3}{|c|}{ MYOBASE.ORG } \\
\hline \multicolumn{3}{|c|}{ LE PORTARL DOCUMENTAIRE SUR LES MALADIES NEUROMUSCULARRES } \\
\hline \multicolumn{3}{|c|}{$\begin{array}{l}\text { Ce portail documentaire spécialisé dans le domaine des maladies } \\
\text { neuromusculaires, les situations de handicap qu'elles génèrent et leurs aspects } \\
\text { psychologiques est publié par VAFM-Téléthon. }\end{array}$} \\
\hline UN ACCES facle et simple & Un out ergonomique, UNE & En 2019, Myobase \\
\hline \multirow{2}{*}{$\begin{array}{l}\text { - Utiliser les gullements } \\
\text { pour la rechenche doune } \\
\text { exprestion } \\
\text { "muladie de pompe" }\end{array}$} & Wherer Hes & libre à $75 \%$ des \\
\hline & $\begin{array}{l}\text { - Lainez-vous guider par les } \\
\text { tuterlels }\end{array}$ & $\begin{array}{l}55000 \text { notices } \\
\text { bibliographiques }\end{array}$ \\
\hline $\begin{array}{l}\text { - Utiliser le signe + pour } \\
\text { signifier ET } \\
\text { "imar-4mutation" }\end{array}$ & $\begin{array}{l}\text { - Lancex une recherche ot } \\
\text { affinez votre silection grkice } \\
\text { aus flitres }\end{array}$ & $\begin{array}{l}\text { du fond documentaire } \\
\text { collecté depuis } 1990\end{array}$ \\
\hline \multirow{2}{*}{$\begin{array}{l}\text { - Uviliser le signe - pou } \\
\text { signifier NON } \\
\text { "Duchenne-diagnostic" }\end{array}$} & fout Mrosast & \multirow{5}{*}{ 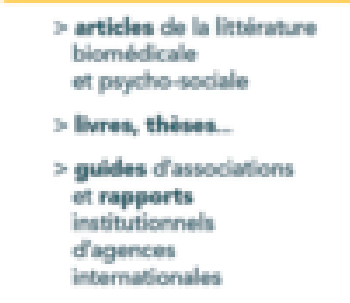 } \\
\hline & 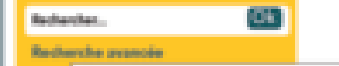 & \\
\hline Priburemenesel & Nen- FitraEs & \\
\hline 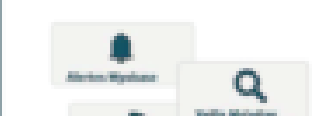 & 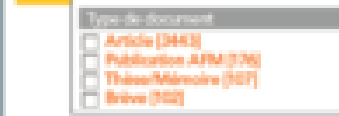 & \\
\hline Di & & \\
\hline $\begin{array}{l}\text { - Clquez sur Fenglet } \\
\text { themutieve qui vous } \\
\text { corwient (haut de la page } \\
\text { draccuel) }\end{array}$ & 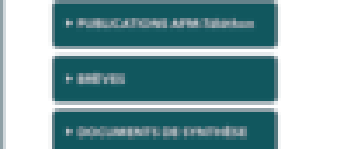 & $\begin{array}{l}>\text { brives en frangals. } \\
\text { gyntheses des articles } \\
\text { midico-scientifiques } \\
\text { internationaus les plus } \\
\text { pertinents }\end{array}$ \\
\hline $\begin{array}{l}\text { - Aborneavous acas alertes } \\
\text { thimutliques. Criber vos } \\
\text { alertes perionnalistes en } \\
\text { ouveant un compte } \\
\text { personnel }\end{array}$ & $\begin{array}{l}\text { - Partagez les nhulhats } \\
\text { de votre recherche }\end{array}$ & $\begin{array}{l}\text { publications } \\
\text { AFM-Tbletthon } \\
\text { devitindes aux } \\
\text { professionels de } \\
\text { santé eu aux personnes } \\
\text { atteintes de mulade. }\end{array}$ \\
\hline $\begin{array}{l}\text { - THechargez la Veillo } \\
\text { Neurommeculatio } \\
\text { - Abormezvous wax fluax RSs }\end{array}$ & $\begin{array}{l}\text { - Utilisea les onglets } \\
\text { thimatiques ou type } \\
\text { de publication }\end{array}$ & $\begin{array}{l}\text { anteintes de malade } \\
\text { neuromaculaine et } \\
\text { b leur entourage }\end{array}$ \\
\hline
\end{tabular}

